median OI of $\mathrm{x}$ in the first day of life. iNO improved OI in all cases, although there was no significant effect on BPD, IVH or mortality. Our data supports the findings of earlier studies that iNO can improve respiratory function in PPHN associated with PPROM. Further prospective trials are needed to assess long term outcomes in this subsection of babies with severe respiratory failure following preterm delivery.

\section{G131(P) MICROBIOLOGICAL FLORA AND THEIR SENSITIVITIES TO ANTIBIOTICS, IN A TERTIARY NEONATAL UNIT AT NORTH EAST OF ENGLAND}

${ }^{1} \mathrm{H}$ Dumelow, ${ }^{2} \mathrm{M}$ Narayanan, ${ }^{1} \mathrm{~N}$ Athiraman. ${ }^{1}$ Neonates, Newcastle Hospitals NHS Foundation Trust, Newcastle-Upon-Tyne, UK; ${ }^{2}$ Microbiology, Newcastle Hospitals NHS Foundation Trust, Newcastle-Upon-Tyne, UK

\subsection{6/archdischild-2015-308599.129}

Background Sepsis remains one of the biggest causes of neonatal morbidity and mortality. The antibiotic guidelines used on our NICU are influenced heavily by national epidemiological data from NeonIN, collected from 2006-2008. The objective of this service improvement project was to establish the current epidemiology of bacteraemia-causing pathogens on our unit, and their sensitivity to our empirical antibiotic regimens, in order to establish whether our guidelines remain adequate.

Methods Laboratory data on positive blood cultures from April 2011 to March 2014 were analysed. We established the common pathogens, their incidence, and their sensitivity to recommended empirical antibiotics.

Results Out of 2367 blood cultures analysed, 116 (5\%) were positive for an organism. These were made up of $70(60 \%)$ Coagulase Negative Staphylococcus, 13 (11\%) Group B Streptococcus, $12(10 \%)$ coliforms, 8 (7\%) Enterococcus faecalis and 6 (5\%) Staphylococcus aureus. The remaining were rarer Gram positive organisms and 1 Haemophilus influenzae. 100\% of organisms that commonly cause early-onset sepsis (GBS and Escherichia coli $\mathrm{n}=19$ ) were sensitive to the recommended antibiotic combination of penicillin and gentamycin. 100\% of non- CoNS pathogens were sensitive to the Amoxicillin and/or Gentamycin in the late onset sepsis regimen of Amoxicillin/Flucloxacillin/Gentamycin. Sensitivity to Flucloxacillin was measured at $22 \%$ of positive cultures tested. Only 54\% of CoNS was tested for sensitivity to Vancomycin, which is the recommended antibiotic for suspected CoNS (eg. central line in situ). 100\% of these were sensitive.

Conclusions Initial analysis shows that guidelines are appropriate for early-onset sepsis and non-CoNS late-onset sepsis. However, the data questions the need for Flucloxacillin in the LOS regimen. There is apparent inconsistency in testing of CoNS for sensitivity to Vancomycin, not allowing a full judgement as to whether this provides adequate cover. The need for Ceftazidime (recommended with Vancomycin when a baby remains sick despite the Amoxicillin/Flucloxacillin/Gentamycin regimen) is questioned by this study.

\section{G132(P) IMPACT OF IMPLEMENTING NATIONAL GUIDELINES ON 'ANTIBIOTICS FOR EARLY-ONSET NEONATAL INFECTION' ON A LEVEL 2 NEONATAL UNIT}

E Liew, A Moylan, T Wickham. Starlight Neonatal Unit, Barnet Hospital, Royal Free London NHS Foundation Trust, London, UK
Aims NICE projected cost savings of $£ 50$ million per annum with its guideline on "Antibiotics for early-onset neonatal infection'. We assessed the impact of implementing this guideline in a Level 2 Neonatal Unit.

Method Retrospective case notes review of neonates born in our hospital who received antibiotics within $72 \mathrm{~h}$ of birth. We compared a pre-guideline implementation cohort (March-April 2012) and a post-guideline cohort (March-April 2013). Data on characteristics of the neonates, reasons for starting antibiotics, length of antibiotics course and adherence to the guidelines were collected.

Results 138 neonates were identified, 57 in the pre-guideline cohort and 81 in the post-guideline cohort (Figure 1). From the pre-guideline cohort, 39 were included. From the post-guideline cohort, 59 were included. The cohorts were matched. Overall, post-guideline implementation, there is a $51 \%$ increase in the number of neonates receiving antibiotics, with the main reason being for neonatal signs and symptoms in both cohorts $(72 \%$ and $77 \%$ ) (Figure 2). However, there was a $9 \%$ rise in neonates being started on antibiotics because of maternal risk factors alone in the post guideline cohort. This is due to the increasing number of mothers started on intravenous antibiotics for suspected invasive bacterial infection, although the criteria defining 'suspected invasive bacterial infection' are unclear. Post-guideline implementation, the number of neonates receiving more than

\begin{tabular}{|c|c|c|}
\hline & $\begin{array}{c}\text { Pre NICE Cohort } \\
2012\end{array}$ & $\begin{array}{c}\text { Post NICE Cohort } \\
2013\end{array}$ \\
\hline $\begin{array}{c}\text { Gestation (median week) } \\
\text { Term (percentage) }\end{array}$ & 38 & 38 \\
\hline $\begin{array}{c}\text { Started antibiotics within } \\
\text { first 24 hours of life } \\
\text { (percentage) }\end{array}$ & 56 & 55 \\
\hline $\begin{array}{c}\text { Admitted to the NNU } \\
\text { (percentage) }\end{array}$ & 79 & 81 \\
\hline
\end{tabular}

Abstract G132(P) Figure 1 Characteristics of the two cohorts

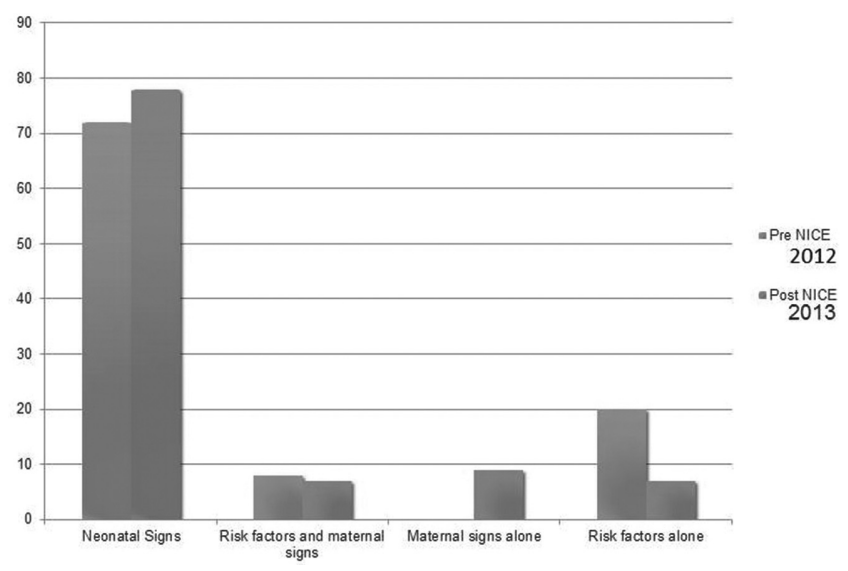

Abstract G132(P) Figure 2 Reason for starting antibiotics

\begin{tabular}{|l|l|l|}
\hline & \multicolumn{2}{c|}{$\begin{array}{l}2012 \\
\mathrm{~N}=39\end{array}$} \\
\hline Up to 2 days & $22(56 \%)$ & $19(32 \%)$ \\
\hline $3-5$ days & $16(41 \%)$ & $28(47 \%)$ \\
\hline $6-7$ days & $1(2.5 \%)$ & $10(17 \%)$ \\
\hline$>7$ days & 0 & $2(3 \%)$ \\
\hline
\end{tabular}

Abstract G132(P) Figure 3 Duration of antibiotics 


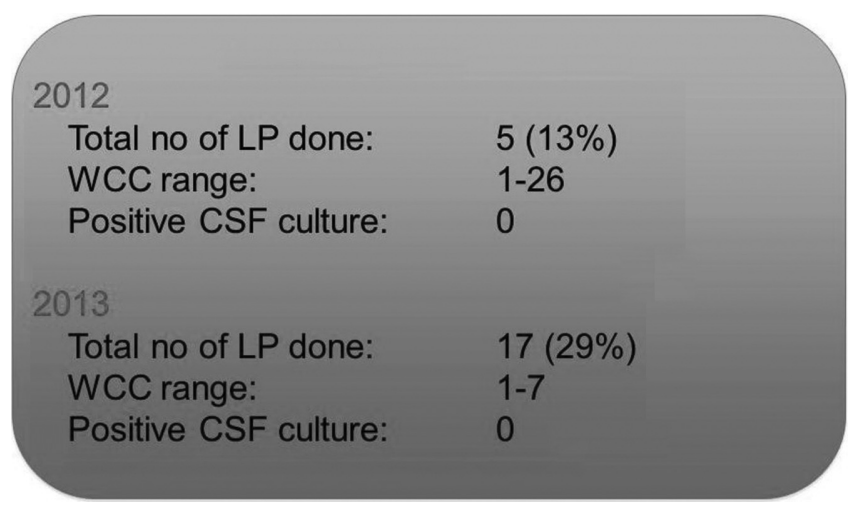

Abstract G132(P) Figure 4 Lumbar puncture

$48 \mathrm{~h}$ of antibiotics increased from $44 \%$ to $68 \%$, due to our microbiology laboratory operating on a $48 \mathrm{~h}$ reporting system for specimen cultures as opposed to $36 \mathrm{~h}$ as suggested by NICE (Figure 3). More neonates with negative blood cultures but with elevated C-reactive protein (CRP) were receiving longer courses of antibiotics. Performing lumbar puncture if the CRP $>10 \mathrm{mg} / \mathrm{L}$ resulted in a $16 \%$ increase in the number performed, with no significant clinical impact (Figure 4).

\section{Conclusion}

Implementation of NICE guidelines in our unit has resulted in increased cost due to more neonates receiving antibiotics for longer duration. In order to achieve NICE's cost saving projections, further clarification on criteria for starting maternal intravenous antibiotics is needed, as is a clearer definition of 'strong suspicion of sepsis' in neonates with negative blood culture, and a change in hospital laboratory reporting protocol.

\section{G133(P) PERSISTENT PULMONARY HYPERTENSION IN NEWBORNS FOLLOWING PRETERM RUPTURE OF MEMBRANES- A CASE SERIES}

${ }^{1} \mathrm{G}$ Campbell, ${ }^{2} \mathrm{R}$ Metcalfe, ${ }^{1} \mathrm{~A}$ Powls, ${ }^{2} \mathrm{~F}$ Mackenzie, ${ }^{3} \mathrm{~S}$ Lip. ${ }^{1}$ Neonatal Unit, Princess Royal Maternity Hospital, Glasgow, UK; ${ }^{2}$ Maternity Unit, Princess Royal Maternity Hospital, Glasgow, UK; ${ }^{3}$ Neonatal Unit, Southern General Hospital, Glasgow, UK

\subsection{6/archdischild-2015-308599.131}

Historically it has been understood that babies born after a prolonged period of oligohydramnios, secondary to PROM at an early gestation, have pulmonary hypoplasia which leads to severe respiratory failure. We present a case series of infants with a history of PROM who presented in the first few hours of life with severe respiratory failure and were treated with iNO. The clinical response to iNO suggests that the underlying cause of the respiratory failure is predominantly PPHN rather than pulmonary hypoplasia.

13 infants with Gestational age range 26-29 weeks had SROM ranging from 15-27 weeks gestation. Median duration of ROM was 39 days. In the nine cases in whom antenatal information was available, four had a history of oligohydramnios and four had anhydramnios. In five there was evidence of chorioamnionitis on placental pathology, of whom four had clinical chorioamnionitis prior to delivery.

Oxygenation index (OI) just prior to iNO ranged from 112552 and the reduction in OI after starting iNO can be seen in Figure 1. There is no difference in days on ventilation when compared to gestation matched controls without PROM, but they appear to require supplementary oxygen for longer.

This case series demonstrates a marked improvement in hypoxic respiratory failure in babies with PPROM and oligohydramnios (regardless of gestation), providing evidence that the

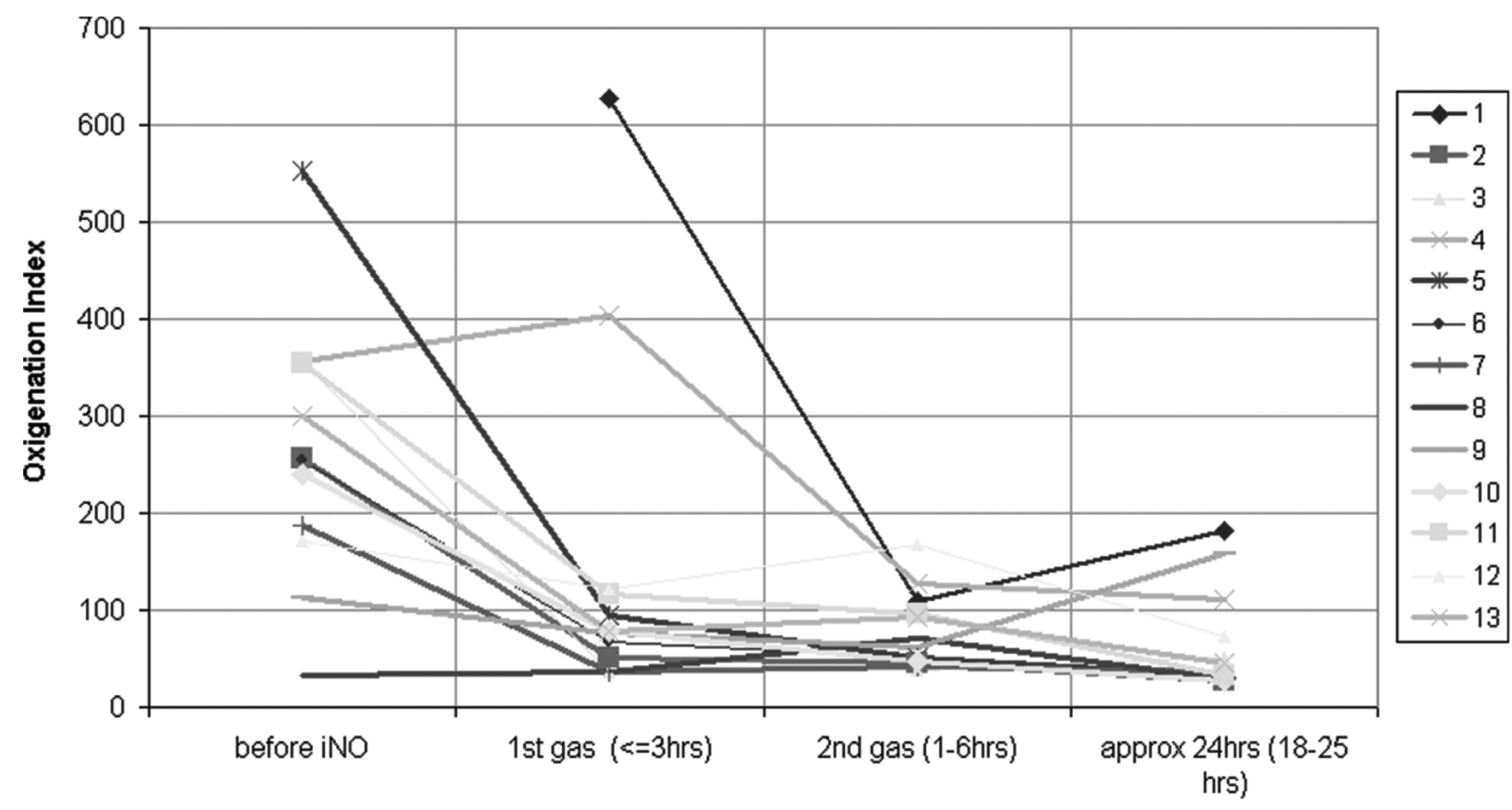

Administration of NO 